\title{
Noblesse et croisade à la fin du Moyen Âge
}

Jacques Paviot

\section{(2) OpenEdition}

Journals

Édition électronique

URL : https://journals.openedition.org/crm/751

DOI : 10.4000/crm.751

ISSN : 1955-2424

Éditeur

Honoré Champion

Édition imprimée

Date de publication : 30 décembre 2006

Pagination : 69-84

ISSN : 1272-9752

Référence électronique

Jacques Paviot, « Noblesse et croisade à la fin du Moyen Âge », Cahiers de recherches médiévales [En ligne], 13 | 2006, mis en ligne le 27 novembre 2009, consulté le 15 décembre 2022. URL : http:// journals.openedition.org/crm/751 ; DOI : https://doi.org/10.4000/crm.751 


\section{RM}

\section{Noblesse et croisade à la fin du Moyen Âge}

Par son essence même - reprenons la définition donnée par Jean Richard : «une expédition, essentiellement militaire, assimilée par la papauté à une œuvre méritoire et dotée par elle de privilèges spirituels accordés aux combattants et à ceux qui participent à leur entreprise (...) ceux qui prenaient la route de l'Orient et plus spécialement des Lieux saints », mais encore "pour d'autres opérations lancées à l'intérieur de la chrétienté contre des hérétiques ou des ennemis de l'Église de Rome, aussi bien qu'aux frontières de cette même chrétienté contre des païens ou des infidèles ${ }^{1} »-$, la croisade se trouvait réservée en priorité à l'état des combattants, qui est devenu la noblesse. En France, cela a été en quelque sorte institutionnalisé par son intégration dans l'aide aux quatre cas.

À la fin du XIII ${ }^{e}$ siècle, les champs de lutte contre l'infidèle se fixèrent plus ou moins pour deux siècles. Dans la péninsule Ibérique, les Portugais et les Aragonais avaient achevé leur reconquista, tandis que les Castillans vécurent sous un régime de trêves avec le royaume de Grenade qui fut finalement conquis en 1492. À partir de 1415, les Portugais passèrent de l'autre côté du détroit de Gibraltar, au Maroc. À l'extrémité orientale de la Méditerranée, les établissements latins étaient tombés aux mains des Mamelouks, tandis que les Grecs avaient récupéré leur empire. Seul subsistait le royaume chrétien (latin) de Chypre et plus à l'ouest les Hospitaliers s'installèrent dans l'île de Rhodes en 1306-1307. Au nord-est de l'Europe, les chevaliers teutoniques avaient ouvert un nouveau front contre les païens depuis le second quart du $\mathrm{XIII}^{\mathrm{e}}$ siècle. L'originalité du $\mathrm{XIV}^{\mathrm{e}}$ siècle fut l'existence des royaumes turcs d'Anatolie qui furent dominés ensuite par les Ottomans. Bien que la raison première de la croisade, la reconquête de la Terre sainte, demeurait mais perdait de son importance, notamment à cause du développement de la puissance turque, la noblesse disposait toujours de champs de lutte contre l'Infidèle à la périphérie et au-delà de l'Europe. D'autre part, il faut relever qu'à l'instar du chevalier normand dont Guillaume le Conquérant avait saisi les terres sous prétexte qu'il était parti combattre les musulmans en Espagne, tout en reconnaissant: «Je ne crois pas qu'il se puisse rencontrer, sous les armes un meilleur chevalier; mais il est inconstant, prodigue et passe son temps à courir à travers pays $»^{2}$, bon nombre de nobles se passèrent de l'appel ou de l'autorisation ecclésiastique pour partir en croisade.

Pour inciter les nobles à partir en expédition contre les infidèles ou les païens, il fallait garder vivace chez eux l'esprit de croisade, ce à quoi s'attachèrent des auteurs, aussi bien clercs que laïcs. On trouvait toujours dans les bibliothèques l'Historia rerum in partibus transmarinis gestarum de Guillaume de Tyr, avec ses continuations, qui fut traduite en français, anglais, italien, castillan, ainsi que dans une moindre mesure la Conquête de Constantinople de Geoffroy de Villehardouin et l'Histoire de l'empereur Henri d'Henri de Valenciennes. Il en est de même pour le

\footnotetext{
${ }^{1}$ Histoire des croisades, Paris, 1996, p. 7.

${ }^{2}$ Citation d'Orderic Vidal dans M. Bloch, La Société féodale, Paris, 1968, p. 411.
}

Cahiers de Recherches Médiévales, 13, 2006 
premier cycle de la croisade (Chanson d'Antioche, les Chétifs, Conquête de Jérusalem) avec ses différentes branches, qui glorifie la figure de Godefroy de Bouillon, mais qui fut remanié, romancé et s'éloignant par là de l'esprit de croisade, en 1356 (Chevalier au cygne et Godefroy de Bouillon, Baudouin de Sebourc, Bâtard de Bouillon, Saladin).

Le $X V^{\mathrm{e}}$ et surtout le $\mathrm{XV}^{\mathrm{e}}$ siècles furent friands de ces romans ${ }^{3} \mathrm{~d}^{\prime}$ amours contrariées et d'aventures orientales, fantastiques même, où il n'est plus guère question de croisade qui en forme malgré tout la toile de fond, avec les combats contre les Sarrasins, mais aussi leur conversion - absente des croisades des siècles précédents. Dès la fin du XIII ${ }^{\mathrm{e}}$ siècle, l'Italie offre l'Entrée en Espagne (de Charlemagne et ses paladins). Cette veine carolingienne est poursuivie dans un Roland et une autre Espagne un siècle plus tard, avant de fournir la matière des chefs-d'œuvre d'Andrea da Barberino Guerrin le Mesquin (1409), de Luigi Pulci Morgant le Géant (1460?-1479) et de Matteo Maria Boiardo Roland amoureux (1482-1494), en attendant le Roland furieux de L'Arioste (1520-1532), auxquels nous pouvons ajouter Le Pérégrin de Giacomo Caviceo (v. 1500). La langue d'oc a donné le Livre des aventures de Guillaume de La Barre, dû à la plume d'Arnaut Vidal, en 1318. En Catalogne et en Aragon, deux romans eurent beaucoup de succès : Curial et Güelfa (entre 1435 et 1462) et Tirant le Blanc du chevalier Joanot Martorell (livres I-III), continué par Martí Joan de Galba (livre IV) et publié en 1490. Le roman castillan Amadis de Gaule, réécrit par Garcí Rodriguez de Montalvo vers 1492, bien qu'à la limite de notre propos, eut une large diffusion européenne au $\mathrm{XVI}^{\mathrm{e}}$ siècle.

Dans le domaine de la langue d'oïl, la cour d'Anjou-Provence a donné naissance, dans la première moitié $\mathrm{du} \mathrm{XV}^{\mathrm{e}}$ siècle, à Pierre de Provence et la belle Maguelonne qui fut fort prisé en Allemagne, à Paris et Vienne du Marseillais Pierre de La Cépède, puis au Petit Jean de Saintré d'Antoine de La Sale, qui a l'originalité d'aller se battre en Prusse. À la cour de Savoie, Perrinet Dupin commit un Philippe de Madien (1448), tandis que'à celle d'Orléans (?), un auteur anonyme rédigeait Clériadus et Méliadice (1435-1445), en utilisant en partie le roman de Ponthus et Sidoine peut-être dû à la plume de Geoffroy de La Tour Landry (fin $\mathrm{XIV}^{\mathrm{e}}$ s. - début $\mathrm{XV}^{\mathrm{e}}$ s.). C'est la cour de Bourgogne qui produisit le plus ce genre de romans, en reprenant des textes antérieurs ou en faisant œuvre originale, et puisant dans la mémoire des possessions septentrionales ${ }^{4}$ : Histoire d'Hélène reprenant La Belle Hélène de Constantinople du XIV ${ }^{\mathrm{e}}$ siècle, Charles Martel, Chroniques et conquêtes de Charlemagne, Gillion de Trazegnies, Gilles de Chin, mise en prose d'un poème du XIV ${ }^{\mathrm{e}}$ siècle, Jean d'Avesnes, La Fille du comte de Ponthieu, Saladin, Le Comte d'Artois, Louis de Gavre, Les Trois fils de Rois. La cour de France fait figure de parent pauvre avec le précoce Charlemagne de Girart d'Amiens (av. 1308) et le tardif Messire Charles de Hongrie (1495-1498), dans lequel on discerne des influences angevines. À ma connaissance, on ne trouve pas de tels romans dans les littératures germanique, scandinave ou anglaise des $\mathrm{XIV}^{\mathrm{e}}$ et $\mathrm{XV}^{\mathrm{e}}$ siècles.

${ }^{3}$ Pour un tableau général, cf. Michel Stanesco et Michel Zink, Histoire européenne du roman médiéval. Esquisse et perspectives, Paris (Écriture), 1992.

${ }^{4}$ À ce sujet, je me permets de renvoyer à mon livre Les ducs de Bourgogne, la croisade et l'Orient (fin XIV siècle-XV siècle), Paris (Cultures et civilisations médiévales), 2003, p. 207-227. 
À côté de la littérature, l'art offre peu d'œuvres en ce qui concerne la perpétuation de l'idéal de croisade dans les châteaux et demeures nobles. Le seul décor peint que j'ai pu trouver qui puisse se rapprocher d'un sujet oriental, même pas de croisade, et dont la source reste inconnue, est une fresque dans le palais Noriller à Rovereto, dans la vallée de l'Adige 5 . Dans une des chambres de l'hôtel de Saint-Pol à Paris, Charles V avait fait représenter l'histoire de Charlemagne ${ }^{6}$. Ce dernier faisait partie des Neuf Preux ; avec Godefroy de Bouillon il était une des figures tutélaires de la croisade. On retrouve ces Neuf Preux, au début du $\mathrm{XV}^{\mathrm{e}}$ siècle, en pierre à Pierrefonds pour Louis d'Orléans, en peinture à La Manta, dans le Piémont, château appartenant à Waleran de Saluces, fils bâtard du marquis Thomas III, auteur du Chevalier errant, ou à Castel Roncolo à Bolzano, en tapisserie comme celles conservées au Metropolitan Museum of Art de New York, ou celles que possédait Jean, duc de Berry, tissées de fils d'or et d'argent?

La tapisserie offre en effet plus d'œuvres en rapport avec la croisade. Louis d'Orléans possédait une chambre de l'histoire de Charlemagne ainsi que de celle de saint Louis ${ }^{8}$. Bien qu'inspirée des Antiquités judaïques de Flavius Josèphe et tirée du mystère d'Eustache Marcadé $(\dagger 1440)$, la suite la Vengeance de Notre-Seigneur rappelait au spectateur chrétien le devoir de reprendre Jérusalem?.

À défaut d'une forte incitation par la littérature et l'art, les traités d'éducation à l'usage des nobles engageaient les jeunes damoiseaux à parcourir le monde et à combattre contre l'infidèle pour apprendre le métier des armes. Ainsi Jean Petit, en 1389, à l'intention des Martel de Basqueville en Normandie, dans son traité Les condicions qui sont requises a l'enfant d'un seigneur a estre droit gentil $z^{10}$ :
Ainsi la belle servira
Et, en la servent s'en yra,
Si tost com se pourra armer,
En ces voyages d'oultremer,
Et de Pruce et de Barbarie.
Et pour Dieu ne s'espairgne mie,
Mais a la mort nostre Seigneur
Vengier mette pleine greigneur
Qu'il pourra, selon sa puissance.

${ }^{5}$ Cf. Le vie del Gotico. Il Trentino fra Trecento e Quattrocento. Guida, Trente, 2003, n ${ }^{\circ} 2$, p. 60 et 61.

${ }^{6}$ C. de Mérindol, La Maison des Chevaliers de Pont-Saint-Esprit, t. II : Les décors peints. Corpus des décors monumentaux peints et armoriés du Moyen Âge en France, Pont-SaintEsprit, 2001, p. 330.

${ }^{7}$ Cf. Chefs-d'œuvre de la tapisserie du XIV au XVI siècle, catalogue d'exposition, Paris, Grand Palais, 26 octobre $1973-7$ janvier 1974, n 3 et 4, p. 39-43.

${ }^{8}$ Louis d'Orléans et Valentine Visconti. "Mécénat et politique autour de 1400". Le petit journal de l'expo (sic), 26 juin - 12 septembre 2004, Château de Blois, p. 5.

${ }^{9}$ Cf. Chefs-d'œuvre de la tapisserie..., $\mathrm{n}^{\circ}$ 11-13, p. 61-65 (atelier tournaisien ; conservée à Lyon et Saumur).

${ }^{10}$ Dans Le Livre du champ d'or et autres poèmes inédits, éd. P. Le Verdier, Paris, 1896, p. 124-137. On peut noter que Guillaume VI Martel de Basqueville participa à la croisade d'Alexandrie en 1365 et que son cousin Guillaume Martel est indiqué à celle de Barbarie en 1390 (cf. les références infra). 
Et qu'il soit en la gouvernance

D'un expert et bon chevalier

Pour besoingnier et adrecier,

Qui bien saiche le tour des guerres

De pluseurs pars d'estranges terres ;

Et que nulle chose ne face,

Mais garde bien qu'il ne raconte

Fors que la pure verité...

ou encore Hue de Lannoy, au milieu du $\mathrm{XV}^{\mathrm{e}}$ siècle, dans son Enseignement de la vraie noblesse $^{11}$, ou Livre de l'estat de noblesse et chevalerie ${ }^{12}$ :

Il est bien seant en temps de paix que jeunes hommes de noble linaige voisent chercer les estranges contrees et facent voyaiges comme de Jherusalem, saincte Katherine, comme saint Jacques et les royaulmes cristiens ; et qui plus est qui s'emploient en guerre contre les Sarrasins et mescreans, car jeune homme ne peut nulle part mieulx apprendre les affaires du monde que par voiaiges et hanter le fait des armes en estranges contrees et païs.

À côté de ces exhortations, l'appartenance à un des ordres de chevalerie qui fleurirent aux $\mathrm{XIV}^{\mathrm{e}}$ et $\mathrm{XV}^{\mathrm{e}}$ siècles ${ }^{13}$ pouvait engager ses membres à combattre pour la défense de la foi chrétienne et de l'Église. Tels furent la compagnie du SaintEsprit au droit désir, ou compagnie du Nœud, de Louis, roi de Naples, en 13521353, l'ordre de l'Épée de Pierre I ${ }^{\mathrm{er}}$ de Lusignan, roi de Chypre, en 1359, l'ordre du Collier d'Amédée VI, comte de Savoie, fondé en 1364 avant son départ en croisade, l'ordre de la Nef de Charles, roi de Naples, en 1381. Le véritable ordre dont le seul but était la croisade fut celui rêvé toute sa vie par Philippe de Mézières (1327 ?1405), la Milice de la Passion de Jésus-Christ, qu'il a proposé à partir de $1367^{14}$. Pour ceux qui avaient la vocation, il était toujours possible d'entrer dans un des ordres militaires: Hôpital de Saint-Jean de Jérusalem dans toute l'Europe et en

\footnotetext{
${ }^{11}$ Bruxelles, Bibliothèque royale de Belgique, ms. 10314 ; F. Hachez, «Un manuscrit de l'Enseignement de la vraie noblesse, provenant de la bibliothèque de Charles de Croy, comte de Chimay », Annales du Cercle archéologique de Mons, XXIII, 1892, p. 91-104, l'attribue à Ghillebert de Lannoy; B. Sterchi, «Hugues de Lannoy, auteur de l'Enseignement de vraie noblesse, de l'Instruction d'un jeune prince et des Enseignements paternels », Le Moyen Âge, CX, 1/2004, p. 79-117, plus justement à son frère.

${ }_{12}$ Bibliothèque nationale de France, ms. fr. 1227 (copie du 2 avril 1496), d'où la citation $\left(\mathrm{f}^{\circ} 55^{\vee}\right)$, publiée dans P. Contamine, La Noblesse au royaume de France de Philippe le Bel à Louis XII. Essai de synthèse, Paris, 1997, p. 186.

${ }^{13}$ Pour les ordres royaux, cf. D'A. J. D. Boulton, The Knights of the Crown. The Monarchical Orders of Knighthood in Later Medieval Europe 1325-1520, Woodbridge - New York, 2 éd. 2000 ; J. Paviot, "Les ordres de chevalerie à la fin du Moyen Âge », Bulletin de la Société nationale des antiquaires de France, 2001, p. 195-205.

${ }^{14}$ Un certain nombre de nobles et de non nobles s'étaient cependant engagés dans cette Milice, soit pour devenir chevaliers, soit pour la soutenir, de France et d'Angleterre surtout, mais aussi de Castille, d'Aragon, de Gascogne, de Navarre, d'Allemagne et d'Écosse ; cf. A. Molinier, «Description de deux manuscrits contenant la règle de la Militia Passionis Jhesu Christi de Philippe de Mézières », Archives de l'Orient latin, I, 1881, p. 362-364.
} 
Méditerranée orientale pour la guerre contre les Sarrasins et les Turcs, Sainte-Marie des Teutons en Prusse, et en péninsule Ibérique: Saint-Georges d'Alfama en Catalogne, Montesa dans le royaume de Valence (ces deux ordres étant unis en 1400), Calatrava, Alcantara, Saint-Jacques de l'Épée en Castille, Avis, Saint-Jacques de l'Épée, et Christ au Portugal ${ }^{15}$. Certaines familles envoyaient ainsi régulièrement des fils dans un ordre militaire, tels les Bauffremont, famille du duché de Bourgogne $^{16}$, avec l'ordre de l'Hôpital. À la fin du XIV ${ }^{\mathrm{e}}$ siècle, Pierre, fils de Philibert et d'Agnès de Jonvelle, se trouvait à Rhodes ${ }^{17}$. Une cinquantaine d'années plus tard, un cousin de la branche cadette, Érard, fils d'Henri et de Jeanne de Vergy ${ }^{18}$ et frère du plus célèbre Pierre, comte de Charny, était commandeur de Bellecroix et s'était «grandement employéz pour la deffense de la foy cristienne »"

Certaines familles avaient un ancêtre, réel ou imaginaire, qui était lié à la croisade ou à la lutte contre les Sarrasins : ainsi les comtes de Genève disaient descendre d'Olivier, le paladin de Charlemagne, les Clèves du Chevalier au cygne, grand-père de Godefroy de Bouillon. Le choix des prénoms, pour lequel il faudrait une étude, pourrait indiquer une vivacité de l'esprit de croisade. Comment ont été donnés les noms des chefs de la Première croisade, Godefroy, Baudouin, Raymond, Bohémond, et pour la péninsule Ibérique Alphonse ou Henri, sans parler de Jacques, Matamoros, «le tueur de Maures»?

Pour les ancêtres réels, nulle famille ne pouvait égaler celle de France, avec, en ligne continue, Louis VII (Deuxième croisade, 1147-1149), Philippe Auguste (Troisième croisade, 1190-1191), Louis VIII (croisades contre les Albigeois, 1215, 1219, 1226), Louis IX (1247-1254; 1270), Philippe III le Hardi («croisade d'Aragon », 1285) ; Philippe IV le Bel prit la croix avec ses fils en 1313, Philippe VI de Valois en 1333, Jean II le Bon en 1362 ; Charles VI, dans le but de la paix et de la réunion de l'Église, projeta une croisade conjointe avec Richard II d'Angleterre en 1396 ; enfin Charles VIII partit en 1494 pour conquérir Naples, Constantinople et Jérusalem. Dans la branche cadette de Valois-Bourgogne, Philippe le Hardi aurait pris la croix avec son père en 1362, il aurait dû partir en 1395, soit en Prusse, soit en Hongrie où son fils Jean, comte de Nevers (plus tard sans Peur), alla l'année suivante ; Philippe le Bon fit le vœu de la croisade en 1451, réitéré au banquet du Faisan en 1454, avec son fils Charles.

On pourrait croire qu' aux $\mathrm{XIV}^{\mathrm{e}}$ et $\mathrm{XV}^{\mathrm{e}}$ siècles il y avait de l'affectation chez ces rois ou princes : en Hongrie, la croisade fut régulièrement proclamée en 1314, $1325,1332,1335,1352$ et 1354, en Pologne en 1325, 1340, 1343, 1351, 1354, 1355, 1363 et 1369 (les choses devinrent plus sérieuses avec l'apparition des Ottomans à

${ }^{15}$ Cf. A. Demurger, Chevaliers du Christ. Les ordres religieux-militaires au Moyen Âge $\left(X I^{e}\right.$ XVI $I^{e}$ siècle), Paris, 2002.

${ }^{16}$ Cf. M.-T. Caron, La Noblesse dans le duché de Bourgogne, 1315-1477, Lille (Économies et sociétés), 1987, p. 315-356.

${ }^{17}$ Il apparaît dans un tableau généalogique dans l'ouvrage cité note précédente (p. 344) ; cf. J.C. Poutiers, «Les Chevaliers de Rhodes à la bataille de Nikopol (1396)», Études balkaniques, 1, 1981, p. 97-99 ; J.-M. Roger, «Ayme d'Oiselay, prieur d'Aquitaine, et sa brigue du prieuré de France en $1416 »$, Bulletin de la Société des antiquaires de l'Ouest et des Musées de Poitiers, $5^{\mathrm{e}}$ série, VI, 1992, p. 116 et 124.

${ }^{18}$ Absent des tableaux généalogiques cités.

${ }^{19}$ J. Paviot, Les ducs de Bourgogne, la croisade et l'Orient..., p. 251 (1447). 
la fin du siècle). Pourtant des expéditions furent organisées et un certain nombre eurent effectivement lieu, permettant aux nobles d'Europe latine d'y participer. Pour la Méditerranée orientale, il y a eu la tentative de Charles de Valois pour la récupération de l'empire de Constantinople en 1307 et 1308, la "croisade des Hospitaliers » en 1310, la ligue navale formée de Venise, l'Hôpital, Constantinople, la papauté et la France qui s'empara de Smyrne en $1344^{20}$, la croisade d'Humbert, dauphin de Viennois, en 1345-1347, celle de Pierre ${ }^{\text {er }}$ de Chypre à la suite d'un voyage dans l'Europe latine, qui fit le sac d'Alexandrie en 1365, celle d'Amédée VI, comte de Savoie, en Thrace et Bulgarie en 1366, celle de Barbarie, dirigée par Louis, duc de Bourbon, en 1390, avec l'échec du siège de Mahdia, celle de Nicopolis en 1396, la défense de Constantinople assiégée par les Ottomans en 13981400, le secours de Chypre en 1426 , de Rhodes en 1440 et 1444 , la croisade de Varna en 1444, la défense de Constantinople en 1453 (par les Italiens), la ligue dirigée par Venise en 1472, la défense de Rhodes en $1480^{21}$.

Les Teutoniques étaient allés dès 1225 en Europe du nord-est et y ont installé leur siège en 1309, à Marienbourg. De là, ils menèrent une croisade perpétuelle contre les païens au moyen de reise annuelles dans lesquelles ils accueillirent la chevalerie occidentale, jusqu'à la défaite de Tannenberg en $1410^{22}$. Dans la péninsule Ibérique, il y eut la grande victoire de Salado en 1340, les longs sièges d'Algéciras en 1342-1344 et de Gibraltar en 1349 et 1350, puis un régime de trêves entrecoupées de petites expéditions avant la reprise de la Reconquista en 1482 jusqu'à la conquête de Grenade en 1492. En 1415, les Portugais ouvrirent un nouveau front au Maroc avec la prise de Ceuta. En 1437, ils échouèrent à prendre Tanger. À la seconde génération, le roi Alphonse V reprit l'offensive et s'empara d'Alcacer Seguer en 1458, et surtout de Tanger et d'Arzila en 1471.

Ces différentes expéditions ont rythmé les départs en croisade des nobles européens. En quel nombre y ont-ils participé ? Des listes ont été établies pour les croisades d'Alexandrie en 1365 par Joseph Delaville Le Roulx ${ }^{23}$, de Barbarie en 1390 par Léon Mirot ${ }^{24}$ et de Nicopolis en 1396 par Joseph Delaville Le Roulx et Aziz Suryal Atiya ${ }^{25}$ et ont été reprises par ce dernier dans son livre The Crusade in the Later Middle Ages ${ }^{26}$. En gros, on a quatre-vingts noms de nobles pour la croisade

${ }^{20}$ Confiée aux Hospitaliers en 1374, reprise par les Turcs en 1402.

${ }^{21}$ Cf. A. S. Atiya, The Crusade in the Later Middle Ages, Londres, 1938 ; N. Housley, The Later Crusades, 1274-1580. From Lyons to Alcazar, Oxford, 1992.

${ }^{22}$ Cf. E. Christiansen, The Northern Crusades. The Baltic and the Catholic Frontier 11001525, Londres-Basingstoke, 1980; W. Paravicini, Die Preussenreisen des Europäischen Adels, t. I et II, Sigmaringen (Beihefte der Francia, 17/1-2), 1989 et 1995 ; M. Paravicini poursuivant ses recherches et ses publications à ce sujet, je ne m'intéresserai pas ici à la croisade de Prusse.

${ }^{23}$ La France en Orient au XIV siècle. Expéditions du maréchal Boucicaut, t. II, Paris (Bibliothèque des Écoles françaises d'Athènes et de Rome, 45), 1886, p. j. III, p. 12-13.

${ }^{24}$ «Une expédition française en Tunisie au XIV ${ }^{\mathrm{e}}$ siècle. Le Siège de Mahdia (1390) », Revue des études historiques, $97^{\mathrm{e}}$ année, 1931, appendice, p. 396-406.

${ }^{25}$ La France en Orient au XIV siècle..., p. j. XXII, p. 78-86; The Crusade of Nicopolis, Londres, 1934, app. VI, p. 144-148.

${ }^{26}$ Londres, 1938, app. IV, p. 517-528. Les historiens auraient pu faire de même pour les croisades d'Humbert II en 1345 et d'Amédée VI en 1366, vu les sources publiées; il est vrai que leur recrutement était plus limité. J'ai essayé d'en dresser une comparative, à partir des 
d'Alexandrie, deux cent trente pour celle de Barbarie, et deux cent quatre-vingt cinq pour celle de Nicopolis. On remarque d'emblée une importante mortalité dans les deux dernières, due aux maladies qui sévisssaient dans le camp des assiégeants de Mahdia et à l'hécatombe qu'ont fait subir les Ottomans aux croisés à la suite de la défaite de Nicopolis.

Le recrutement de ces croisades, surtout celles d'Alexandrie et de Nicopolis, était européen, mais à cause des sources nous trouvons principalement des nobles français. Pourtant quelques noms étrangers apparaissent. En 1365, les croisés étaient «Anglais, Chypriotes, Français et Allemands », mais les chroniques n'indiquent que Brunswick, sans doute Otto von Braunschweig-Grubenhagen ${ }^{27}$, le comte de Hereford, un Écossais anonyme ${ }^{28}$, et deux Italiens, Pierre de Grimani et Guillelmo Visconti. Des études plus récentes permettent de jeter un peu de lumière sur la participation anglaise ${ }^{29}$. Durant son tour d'Europe, en 1363, Pierre $\mathrm{I}^{\mathrm{er}}$ de Chypre avait rencontré Édouard III en Angleterre, puis le prince de Galles en France. Si Édouard III ne s'engagea pas, il laissa aller ses nobles d'Angleterre et de Gascogne. Ainsi John de Grey de Codnor, Miles de Stapleton de Bedale, Stephen Scrope, John de Argentine, Nicolas Sabraham, se trouvèrent à la prise d'Alexandrie, mais, selon la rumeur, les Anglais furent responsables de la perte de la ville, se retirant après avoir fait un gros butin. Cependant, certains de ceux-ci, tels Nicolas Sabraham, John de Grey, Miles de Stapleton restèrent en Orient jusqu'en 1367 et allèrent à Adalia en Anatolie, attaquèrent Tripoli, brûlèrent Jaffa, défendirent Gorighos. D'autres, comme le Gascon Florimont de Lesparre se joignirent entre-temps à la croisade d'Amédée VI de Savoie à Constantinople et en Bulgarie, en 1366. Quant à Humphrey de Bohun, comte de Hereford, Norhtampton et Essex, connétable d'Angleterre et chevalier de l'ordre de la Jarretière, il n'alla en Orient qu'en 1367 à la tête d'une troupe d'hommes d'armes anglais et participa aux opérations de cette année-là.

La croisade de Barbarie ayant été transportée par des Génois, il est normal d'en retrouver, mais là encore deux seuls sont cités: Giovanni Centurione, dit Oltramarino, et Antonio Marco ; on relève aussi un Robert de Pouille. De Catalogne venait le vicomte de Rosas. On trouve aussi un petit nombre d'Anglais, dont certains étaient membres de l'hôtel royal : John Beaufort, le plus important, mais aussi John

dons des ducs de Bourgogne : cf. «La croisade bourguignonne aux $\mathrm{XIV}^{\mathrm{e}}$ et $\mathrm{XV}^{\mathrm{e}}$ siècles, un idéal chevaleresque? », à paraître dans Francia.

27 Cependant W. Paravicini, «Fürstliche Ritterschaft: Otto von BraunschweigGrubenhagen », Braunschweigische Wissenschaftliche Gesellschaft. Jahrbuch 1994, p. 97138 , ne mentionne pas sa participation à la croisade.

${ }^{28}$ Sans doute Norman Leslie ; cf. A. Macquarrie, Scotland and the Crusades, 1095-1560, Édimbourg, 1985, p. 81-84 (il était accompagné de son frère Walter).

${ }_{29}$ T. Jones, Chaucer's Knight. The portrait of a medieval mercenary, Londres, 1980 ; M. Keen, "Chaucer's Knight, the English Aristocracy and the Crusade », English Court Culture in the Later Middle Ages, éd. V. J. Scattergood et J. W. Sherbone, Duckworth, 1983, p. 45-61 ; C. Tyerman, England and the Crusades, 1095-1588, Chicago-Londres, 1988, p. 288-293 ; A. Luttrell, «English Levantine Crusaders, 1363-1367 », Renaissance Studies, II2: A Tribute to Denys Hay, 1988, p. 143-153; Id., "Chaucer's Knight and the Mediterranean », Library of Mediterranean History, ed. Victor Mallia-Milanes, t. I, Malte, 1994, p. 127-160. 
Russell, Henry Scrope, John Clanvow, William Neville, un Courtenay (peut-être le comte de Devon), John Clinton, John Cornwall, tous chevaliers, et l'écuyer William Fotheringay ${ }^{30}$.

On le voit, il faut compléter les listes établies par Delaville Le Roulx, Mirot et Atiya. Cependant leur étude et leur comparaison peuvent livrer des enseignements intéressants pour la noblesse française. Certains partaient en famille, bien qu'il faudrait des recherches généalogiques plus poussées pour savoir s'il s'agissait de pères et de fils, d'oncles et de neveux, de frères ou de cousins. En 1365, on relève Jean et Jédouin de Beauvilliers, Baudri et Bonau de Bon, Guillaume et Jean de Saulx, des Bourguignons, et surtout les trois Le Baveux, Guy, Renaud et Robert. En 1390, Alain et Jean de Champagne qui y laissèrent tous les deux la vie, ainsi que Fouques et Gautier d'Escaufourt, Aleaume et Aubert de La Mothe, et Guichard et Tristan de La Mouleraye; Charles et Robert d'Hangest, de Picardie, Jean VII, comte d'Harcourt et Robert d'Harcourt, de Normandie, le seigneur de Ligne et Jean de Ligne, de Flandre, Blain Loup et son frère, Louis et Philippe de Poitiers, de Champagne, Jean III, comte de Sancerre, et Étienne de Sancerre, seigneur de Vailly, Guillaume de Vienne, seigneur de Saint-Georges, et Jean de Vienne, amiral de France, du comté de Bourgogne. Parmi ceux qui sont venus à trois, Hugues, seigneur de Chastellus, et ses deux fils Jean (de Chateaumorand, qui alla dix ans plus tard à Constantinople avec Boucicaut) et Guichard, du Bourbonnais ${ }^{31}$; les Bourguignons Geoffroy, Guillaume dit Sauvage, et Philippot de Jaucourt; les La Trémoille, Guillaume seigneur d'Usson, son frère aîné Guy seigneur de Sully, Jean seigneur de Jonvelle; Jean, Jean le Bâtard et Renaud de Trie, les deux premiers y perdant la vie.

Pour la croisade de Nicopolis, la liste est plus grande : les frères Philippe et Henri de Bar, le premier tué à la bataille, le second mort lors du retour; Guillaume et Jean des Bordes, du Beauvaisis; les frères Damas ( $\dagger$ ) et Jacques de Busseul, du Charrolais; Henri de Chalon et Hugues, seigneur d'Arlay ( $\dagger$ ), du comté de Bourgogne; Hugues $(\dagger)$ et Pierre de Chandios, du duché de Bourgogne; Jean de Coligny, seigneur de Cressia, et son fils aîné Jacques ( $\dagger$ ), entre comté de Bourgogne et duché de Savoie ; Jacques de Courtiambles et son fils ; Huguenin Damas, seigneur de La Bazole, et Joceran ( $\dagger$ ) ; Étienne et Jean ( $\dagger$ ) de Germigny, trois familles de Bourgogne ; Jean et Olivier de Halluin, Bertrand et Roland Le Bruwere, de Flandre ; Godemart et Jean de Linières, d'Anjou; Guillaume et Huguenin de Lugny, du Mâconnais; Jean et Louis Metteneye, de Flandre; Henri de Montbéliard et son beau-frère; Guillaume et Philippot de Nanton, de Bourgogne; Jean et Rasse de Tauques; Jean et Nicolas Uten Hove, de Flandre ; Anceau et Philibert de Villiers, ou Villers, alors de Bourgogne. Les La Trémoille furent de nouveau trois: Guillaume, seigneur d'Usson, son fils Philippe, et son frère Guy, seigneur de Sully, tous les trois y perdant la vie; d'Artois, il y eut chez les Montcavrel le seigneur, son fils ( $\dagger$ ) et le Borgne ( $\dagger)$, et chez les Renty le Galois, Rasse et le Bâtard ; de Bourgogne, Georges, Girard $(\dagger)$ et Jean de Rigny, et Guillaume de Vergy, son frère et Jacques. Les Bâtards de Flandre vinrent à quatre, Louis, dit le Haze (†), Raoul, Renaut et Victor,

${ }^{30}$ C. Tyerman, England and the Crusades..., p. 279.

${ }^{31}$ Cf. G. Schlumberger, «Jean de Chateaumorand», dans Id., Byzance et les croisades. Pages médiévales, Paris, 1927, p. 281-336. 
ainsi que les Roye, de Picardie : Dreux, dit Lancelot $(\dagger)$, Jean, seigneur de Roye $(\dagger)$, Matthieu son fils, Renaut. Les Vienne, du comté de Bourgogne, vinrent encore plus nombreux : Guillaume, Jacques $(\dagger)$ et Jean de Longwy, Jean, l'amiral de France $(\dagger)$ et son fils $(\dagger)$.

Je n'ai cité que les mêmes noms de famille. En fait, nous pourrions étendre ces participations familiales synchroniques à la croisade. Pour en revenir à la famille Bauffremont mentionnée à propos de l'ordre de l'Hôpital, elle était liée aux familles de Saulx, Vergy, Vienne, Charny et Poitiers ${ }^{32}$. La famille de Vergy était alliée à celles de Chalon et de Vienne ${ }^{33}$. Comme autre exemple plus tardif, on peut citer le cas de Geoffroy de Thoisy, un noble bourguignon qui s'est spécialisé dans la croisade pour le duc Philippe le Bon. En 1444, il est parti à Rhodes et à Constantinople avec son cousin Jacot et son neveu Pierre de Moroges $^{34}$.

La comparaison des listes de croisés de la deuxième moitié du $\mathrm{XIV}^{\mathrm{e}}$ siècle $^{35}$ nous montre qu'un certain nombre a participé à plus d'une expédition, ce qui peut révéler un goût pour la croisade, du moins pour les aventures guerrières lointaines. Les Écossais Norman et Walter Leslie ont effectué le voyage de Prusse en 1356 avant de partir pour Chypre en 1363, peu après la rencontre entre leur roi David et Pierre $\mathrm{I}^{\mathrm{er}}$ de Lusignan ${ }^{36}$. À trente ans d'écart, il est difficile de savoir s'il s'agit de la même personne qui est allé à Alexandrie, en Barbarie ou à Nicopolis, ou d'un fils, d'un neveu ou d'un cousin, tels que Guillaume Martel de Basqueville (1365 et 1390), Renaud de Nantouillet (1365 et 1390) ou Jean de Rochefort (1365 et 1396).

Un certain nombre de nobles ont participé à la croisade de Barbarie et à celle de Nicopolis: Henri d'Anthoing, Philippe d'Artois, comte d'Eu, ainsi que le sénéchal d'Eu, Philippe de Bar, Philippe, dit Sanglier, de Boutervilliers, Geoffroy de Charny, Enguerrand VII de Coucy, Jacques de Courtiambles, Guillaume de Graville, Robin de La Cressonnière, Guillaume de La Trémoille, seigneur d'Usson, Guy de La Trémoille, seigneur de Sully, le seigneur de Lespinasse, celui de Linières, Jean, dit Friant, de Montigny, Hélion de Naillac, Guiot de Roussay, Jean de Roye, Jean de «Siffrevast», Jean de Trémangon, Jean de Trie, Guillaume de Vienne, Jean de Vienne, amiral de France, auxquels on peut ajouter John Beaufort, comte de Somerset, bâtard de Jean de Gand, duc de Lancastre ${ }^{37}$.

Pour savoir si des traditions pouvaient être établies ou s'établir dans les familles, il faut voir si certains de leurs membres ont pris part à des croisades successives. Un exemple précoce est celui de Thibaut de Chepoy, maître des arbalétriers de France, qui dirigea entre 1306 et 1310 l'expédition pour la récupération de l'empire latin de Constantinople en faveur de Charles de Valois. Il y fut en compagnie de son frère Jean et de son fils Jean. Ce dernier joua plus tard, en 1334, un rôle dans la guerre contre les Turcs, commandant le corps français sous les

${ }^{32}$ Cf. les tableaux généalogiques dans M.-T. Caron, La noblesse dans le duché de Bourgogne..., p. 342-345.

${ }^{33}$ Ibid., p. 370.

${ }^{34}$ Cf. J. Paviot, Les ducs de Bourgogne, la croisade et l'Orient..., notamment p. 101 et 107.

${ }^{35}$ Celles reprises dans A. S. Atiya, The Crusade in the Later Middle Ages..., et celle que j'ai établie d'après les dons des ducs de Bourgogne, dans mon article «La croisade bourguignonne aux $\mathrm{XIV}^{\mathrm{e}}$ et $\mathrm{XV}^{\mathrm{e}}$ siècles...».

${ }^{36}$ A. Macquarrie, Scotland and the Crusades..., p. 81.

${ }^{37}$ M. Keen, «Chaucer's Knight... », p. 56 (il alla aussi en Prusse en 1391). 
Vénitiens ${ }^{38}$. Par un procès à propos de leurs armes, nous sommes assez bien renseignés sur la famille Scrope. Dans la branche aînée de Bolton, William et son fils Stephen allèrent en Prusse, en 1394. Dans la branche cadette de Masham, William était dans la compagnie du comte de Hereford à Satalie en 1367. Son neveu homonyme, fils de son frère aîné Henry, était mort en Prusse en 1362. Le frère de ce dernier, Stephen, fut fait chevalier à Alexandrie. Un troisième frère, semble-t-il, Henry, se joignit à la croisade d'Amédée VI, comte de Savoie, mais mourut à Mésembrie ${ }^{39}$.

Pour les expéditions postérieures, il nous faut à nouveau comparer les listes disponibles, à partir desquelles on peut établir le tableau suivant :

\begin{tabular}{|l|l|l|l|}
\hline & ALEXANDRIE 1365 & BARBARIE 1390 & $\begin{array}{l}\text { NICOPOLIS } \\
\mathbf{1 3 9 6}\end{array}$ \\
\hline d'Aunoy & & Robert & Guillaume \\
\hline Boucicaut & & Geoffroy (frère) & Jean (frère) \\
\hline de Boves & & Perrinet & Jean \\
\hline du Bus & Yvonnet & $\begin{array}{l}\text { Gillaume } \\
\text { seigneur) }\end{array}$ \\
\hline de Châtillon & & Gaucher & \\
\hline de Clairvaux & (seigneur) & Eustache ( $\dagger$ ) & Guillaume \\
\hline de Craon & & Amaury ( $\dagger)$ & $\begin{array}{l}\text { Huguenin } \\
\text { Joceran }\end{array}$ \\
\hline de Damas & & Robert & Jean \\
\hline d'Egreville & & Guiot & Tort \\
\hline des Essarts & & Pierre & Charles \\
\hline d'Estouteville & Nicolas, sgr de Torcy & Jean le Jeune & Jacques \\
\hline du Fay & Oiselet & & Jean (fils) \\
\hline de Garencières & & Jean (seigneur) & Jean \\
\hline d'Hangest & & $\begin{array}{l}\text { Charles } \\
\text { Robert }(\dagger)\end{array}$ & \\
\hline de Jaucourt & Philippe & $\begin{array}{l}\text { Geoffroy } \\
\text { Guillaume, dit Sauvage } \\
\text { Philippot }\end{array}$ & \\
\hline de La Heuze & & le Baudrain & Jean ( $\dagger)$ \\
\hline de Mailly & Jacques & Eustache ( $\dagger)$ & \\
\hline $\begin{array}{l}\text { de Saint- } \\
\text { Germain }\end{array}$ & & (seigneur) & Jean \\
\hline & & & \\
\hline
\end{tabular}

Il est remarquable que la famille bourguignonne de Jaucourt, qui avait trois représentants en Barbarie, n'ait envoyé personne à la croisade de Nicopolis, alors que le duc de Bourgogne suscitait l'entreprise, ce qui peut se comprendre pour celle de Mailly qui a perdu Eustache au siège de Mahdia. En ce qui concerne Boucicaut, le gouvernement avait interdit au maréchal Jean d'aller avec le duc de Bourbon en Barbarie: après avoir organisé les joutes de Saint-Inglevert, il s'était rendu en

${ }^{38}$ Cf. J. Petit, «Un capitaine du règne de Philippe le Bel. Thibaut de Chepoy », Le Moyen Âge, X ( $2^{\mathrm{e}}$ sér., I), 1897, p. 224-239 ; C. de La Roncière, Histoire de la Marine française, t. I : Les origines, Paris, 1899 , p. 211-217 et 232-238.

${ }^{39}$ M. Keen, «Chaucer's Knight... », p. 51 et 52. 
Prusse, où alla son frère cadet Geoffroy l'année suivante 1391 et encore en 13921393.

J'ai donné ailleurs ${ }^{40}$ un tableau comparatif des croisés «bourguignons » aux différentes expéditions de la seconde moitié du XIV siècle, dont la Prusse. Pour les autres personnages mentionnés, on sait que parmi les ancêtres d'Antoinette Roger, dame de Beaufort et vicomtesse de Turenne, femme de Jean Le Meingre, dit Boucicaut, maréchal de France, son arrière-grand-père Guillaume II a dû aller en Prusse dans l'hiver 1352-1353, son grand-père Guillaume III l'ayant précédé dans l'hiver 1349-1350, et que son mari y fut en 1384 et dans l'hiver 1390-1391. Les La Trémoille se rendirent aussi beaucoup en Prusse: Guy VI, seigneur de Sully, en 1379-1380, son frère cadet Guillaume, seigneur d'Usson, en 1379-1380 aussi, et en 1389-1390, leur frère cadet Pierre en 1377-1378, 1379-1380, 1390, 1391-1392 et 1394, les fils de Guillaume, Guillaume en 1398-1399 et Philippe en $1394^{41}$.

Nous sommes mal renseignés sur les Français qui allaient combattre les Maures dans la péninsule Ibérique. Ce champ de bataille semble les avoir peu attirés, au contraire des Anglais ou des Allemands, même si Bertrand du Guesclin déclara son intention en 1366 d'aller en guerre contre les Infidèles, à Grenade, au Maroc et à Jérusalem ${ }^{42}$. Dès 1309, des Anglais sollicitaient des indulgences papales à Avignon pour combattre en Espagne. Henri de Grosmont, comte de Derby, et William Montagu, comte de Salisbury, se distinguèrent dans la croisade d'Alphonse XI de Castille en 1343-1344, à la prise d'Algeciras, puis en attaquant la flotte musulmane au large de Ceuta ${ }^{43}$.

Les comtes anglais ont participé à l'expédition castillane, car ils se trouvaient sur place pour une mission diplomatique. En sens inverse, les rois de Castille envoyaient des ambassades pour informer les souverains au-delà des Pyrénées de l'imminence d'une guerre contre les Maures, et attirer des nobles désireux de les combattre. Ainsi, en 1456, un ambassadeur castillan arriva à la cour de France pour annoncer que son roi organisait une puissante expédition contre le roi de Grenade qui avait l'intention de reconquérir l'Espagne avec l'aide du roi de Tunis et des autres rois d'Afrique du nord (ce qui était sans doute une prétention exagérée). Les chevaliers allemands Georg von Ehingen et Jörg von Ramseiden qui se trouvaient là exprimèrent le désir d'y participer. Le roi Charles VII leur offrit une nouvelle armure, un cheval et trois cents couronnes ${ }^{44}$.

Nous sommes assez bien renseignés sur les préparatifs d'un tel voyage par l'exemple du noble écossais James Douglas, même si les circonstances ne sont pas

\footnotetext{
${ }^{40}$ Dans mon article « La croisade bourguignonne aux XIV et $\mathrm{XV}^{\mathrm{e}}$ siècles... ».

${ }^{41}$ Cf. W. Paravicini, , Die Preussenreisen des Europäischen Adels, t. I, tableaux 21 et 22, p. 173 et 174 .

${ }^{42}$ Cuvelier, La Chanson de Bertrand du Guesclin, éd. Jean-Claude Faucon, Toulouse, t. I, CCCLXXVI, p. 217.

${ }^{43}$ M. Keen, «Chaucer's Knight...», p. 48 ; C. Tyerman, England and the Crusades..., p. 276278 ; A. Luttrell, «Chaucer's Knight... », p. 133-136.

${ }^{44}$ Georg von Ehinge, Reisen nach der Ritterschaft, éd. G. Ehrmann, t. I : Edition, Göppingen (Göppinger Arbeiten zur Germanistik, 262-I), 1979, p. 41-43 ; cf. W. Paravicini, « Georg von Ehingens Reise vollendet», Guerre, pouvoir et noblesse au Moyen Âge. Mélanges en l'honneur de Philippe Contamine, dir. J. Paviot et J. Verger, Paris (Cultures et civilisations médiévales, XXII)., 2000, p. 547-588.
} 
ordinaires. En 1329, Robert Bruce l'appela à son lit de mort, lui rappela qu'il avait voué de combattre les ennemis du Seigneur et de la foi chrétienne, une fois ses guerres terminées ; il ne pouvait plus le faire, aussi il le chargea d'emporter son cœur au Saint-Sépulcre. Dans ce but, James Douglas demanda un sauf-conduit à Édouard III, accordé le $1^{\text {er }}$ septembre 1329 pour sept ans, avec une lettre de recommandation pour Alphonse XI de Castille. Le voyage n'avait pas d'itinéraire précis, sur la route de Jérusalem. Le texte du du sauf-conduit indique que James Douglas partait pour la Terre sainte, en secours aux Chrétiens contre les Sarrasins, avec le cœur du roi Robert, sa durée indique la prévision d'une longue absence, la lettre à Alphonse XI la possibilité de combattre contre les Maures de Grenade. James Douglas fit ses préparatifs durant l'automne et l'hiver et rassembla un groupe de chevaliers et d'hommes d'armes. Avant son départ, il donna un banquet pour la fête du saint patron de la famille, le $1^{\text {er }}$ février 1330, fit une donation pieuse à l'abbaye de Newbattle, et alla s'embarquer à Berwick. La première escale fut L'Écluse en Flandre, où le noble écossais s'informa des perspectives de croisade et encourageait qui le voulait à le suivre à Jérusalem; dans ce but, il recevait royalement à bord de son navire. Il apprit finalement la reprise de la guerre entre la Castille et le royaume de Grenade et partit pour Séville. Il participa avec ses hommes au siège de Teba, mais fut tué le 25 août 1330, quelques jours avant la reddition de la place. Sa chair fut enterrée en terre chrétienne et ses os, ainsi que le cœur de Robert Bruce, rapportés en Écosse ${ }^{45}$.

Pour ses dépenses, James Douglas avait sans doute reçu des gratifications du nouveau roi David II. Le financement des croisés reste mal connu. Quelques pistes peuvent être présentés. Jacques, seigneur de Mametz ${ }^{46}$, est sans doute le meilleur exemple du chevalier croisé français de la fin du XIV ${ }^{\mathrm{e}}$ siècle : voyage de Prusse en 1389-1390, croisade de Barbarie en 1390, pèlerinage à Jérusalem en 1393, de nouveau la Prusse en 1394, enfin la croisade de Nicopolis en 1396. Il a pu, en partie, payer ses déplacements grâce aux dons du duc de Bourgogne Philippe le Hardi :

20 novembre 1385 (mandement, Melun) : 100 fr. pour un cheval ;

4 août 1387 (mandement), ${ }^{\text {er }}$ juin 1388 (quittance) : 200 fr.

6 janvier (mandement, Paris), 20 août 1391 (quittance, Hesdin) : 200 fr. d'or (pour les voyages de Prusse et de Barbarie) ;

14 février (mandement, Arras), 10 avril 1394 (quittance) : 200 fr., à son retour du voyage d'outre-mer;

12 janvier (mandement), 14 février 1395 (quittance) : 300 fr. d'or «pour les fraiz et despens que j'avoie faiz ou voiage de Pruce »;

\footnotetext{
${ }^{45}$ A. Macquarrie, Scotland and the Crusades..., p. 73-79.

${ }^{46}$ De La Chesnay-Desbois et Badier, dans leur Dictionnaire de la noblesse ( $3^{\mathrm{e}}$ éd., t. XIII, Paris, 1868), indiquent Mametz en Artois (dép. Pas-de-Calais, arr. Saint-Omer, cant. Aire-surla-Lys), avec comme armes: d'argent, à trois maillets de sable, 2 et 1 . Or les sceaux conservés de Jacques de Mametz présentent trois chevrons échiquetés et en chef un lambel à trois pendants (cf. références infra). (Il y a aussi un Mametz en Picardie, dép. Somme, arr. Péronne, cant. Albert.)
} 
printemps 1396 : 20 fr. seulement, au départ de la croisade de Nicopolis ${ }^{47}$.

Ajoutons que dans une montre de 1387, pour la guerre du Brabant, on le retrouve en compagnie de Louis Dauphin (Prusse, 1391), Renaud de Nantouillet (Alexandrie, 1365 ; Barbarie, 1390) et Jean Prunellé (Prusse, 1385 ?, 1390, 1391 ; Nicopolis, 1396) ${ }^{48}$.

Ces sommes étaient-elles suffisantes pour le défrayer? Nous pouvons faire une comparaison avec celles dépensées par Guy de La Trémoille à son départ pour la croisade de Nicopolis. Je prends uniquement ce qui concerne l'armement et les chevaux: plates $11 \mathrm{fr}$., bacinets, harnais de jambes, garde-bras et autres armures 32 fr., camail, gorgerette, gousset de maille d'acier 12 fr. (ces premières parties se montant à 55 fr.), parement de cotte d'armes, deux bannières, couverture de cheval (de cendal), réfection d'autres bannières et cottes d'armes 46 fr., soit en tout $101 \mathrm{fr}$.; de plus, deux chevaux ont coûté 112 fr. 10 s. t., tandis qu'un troisième n'a coûté que 14 fr. $^{49}$. Les dons ducaux ne couvraient donc qu'une petite partie des dépenses nécessaires pour de telles expéditions et nous ne savons pas en quelle mesure les chevaliers qui partaient pour les expéditions de croisade s'endettaient.

Par les moyens dont ils disposaient personnellement ou par leurs fonctions, certains nobles ont pu mener des opérations de plus grande envergure, notamment maritimes, plutôt que de s'associer à des expéditions collectives en Espagne, en Prusse, en Barbarie ou en Hongrie. Jean II Le Meingre, dit Boucicaut (1366-1421), maréchal de France, en est le premier exemple. En 1384 et 1385, il se rendit deux fois en Prusse. En 1388-1389, il effectua le pèlerinage de Terre sainte où il vint en aide à Philippe d'Artois, comte d'Eu, qui avait été emprisonné. Empêché de participer à la croisade de Barbarie, il retourna une troisième fois en Prusse en 13901391. Au retour de ce voyage, il fut nommé maréchal de France par Charles VI. En 1396, il mena un corps de troupes dans la croisade de Nicopolis où il fut fait prisonnier. Libéré l'année suivante, il fut commis en 1399 chef du détachement que le roi envoyait au secours de Constantinople assiégée par les Ottomans, d'où il ramena l'empereur Manuel II Paléologue afin qu'il demandât lui-même des secours aux puissances latines. En 1401, il fut nommé gouverneur de Gênes. Il s'occupa de la gestion des colonies de la cité maritime et dirigea, en 1403, une expédition navale contre le roi de Chypre Janus qui assiégeait Famagouste. La paix revenue en Chypre, Boucicaut mena des raids contre les Infidèles, Turcs ou Sarrasins : siège de L'Escandelour (Alaya) en Anatolie, projet de prise d'Alexandrie, débarquement à Tripoli de Syrie, descentes et pillages de Batroun, Beyrouth, Saïda et Lattaquié. La croisière se termina par une bataille navale contre les Vénitiens au début de l'automne. Les Génois expulsèrent Boucicaut en 1409 et celui-ci, commettant les

${ }^{47}$ Dijon, Archives départementales de la Côte-d'Or, B 352 (1385 et 1394), B 353 = Pièces scellées 1547 (1391), B 360 (1387-1388), B 11932 (1395), et mon article «La croisade bourguignonne aux $\mathrm{XIV}^{\mathrm{e}}$ et $\mathrm{XV}^{\mathrm{e}}$ siècle... ».

${ }^{48}$ Ibid., B 11752.

${ }^{49}$ Livre de comptes 1395-1406. Guy de La Trémoille et Marie de Sully, éd. L. de La Trémoille, Nantes, 1887, p. 35, 28, 41. 
mêmes erreurs à Azincourt qu'à Nicopolis, subit le même sort, mais cette fois-ci il ne recouvra pas la liberté ${ }^{50}$.

Boucicaut eut comme contemporain Pero Niño, frère de lait d'Henri III, roi de Castille. En 1404, celui-ci lui confia le soin de lutter contre des corsaires castillans en Méditerranée. Parti de Séville, il passa le détroit de Gibraltar et en profita pour longer la côte d'Afrique du nord et y faire une petite descente. Après avoir chassé les corsaires dans les eaux de la Provence, il alla combattre les Maures jusque dans le port de Tunis et tout le long de la côte de «Barbarie» en rentrant à Séville $^{51}$. Citons encore, soixante ans plus tard, le Douaisien Antoine de Payage, «jeusne chevalier sous quarante ans, riche assez de biens, mais plus encore de vertu et de grand los (louange, honneur)», « qui tant se fit cognoistre en Grenade et en Barbarie, par ses haulx faits, que les nations et mesmes les infidèles en exaltoient sa fame (renommée) pour ses mérites » ainsi que le décrit de manière hyperbolique Georges Châtelain dans sa Chronique ${ }^{52}$. Il y était allé peut-être en 1457. En 1464, il commandait une des galères que Philippe le Bon, duc de Bourgogne, envoyait rejoindre la flotte papale à Ancône, mais dont le voyage s'arrêta à Marseille à cause de la mort du pape. Il se livra alors à la course contre les Maures d'Afrique du nord et les chrétiens qui commerçaient avec eux. Après quelques ennuis à Syracuse pour la vente de ses prises, il désira entrer au service des Vénitiens. Attendant leur réponse, il se rendit à Rhodes, rencontra la galéace pèlerine de Venise qui voulut l'attaquer, mais c'est lui qui s'en empara ${ }^{53}$.

L'un des buts de Philippe le Bon, tout au long de son règne (1419-1467) fut la croisade, ce qui permit à certains d'en devenir des spécialistes : ce fut notamment le cas de Geoffroy de Thoisy. En 1432, il fut choisi pour participer au groupe de pèlerins que le duc de Bourgogne envoyait en Terre sainte sous la direction d'André de Toulongeon. Parmi ceux-ci, se trouvait Bertrandon de La Broquière, chargé d'une mission d'espionnage à travers l'Empire ottoman. En 1441, Geoffroy de Thoisy fut commis par Philippe le Bon pour diriger la petite escadre que celui-ci envoyait au secours des Hospitaliers à Rhodes, alors qu'on redoutait une attaque des Mamelouks. Trois ans plus tard, il commandait les navires armés en Provence, partie de la flotte qui devait rejoindre Constantinople pour mener campagne contre les Turcs. Geoffroy de Thoisy alla d'abord à Rhodes, assiégée par les Mamelouks et fut un des héros de la défense contre les assaillants. Il arriva trop tard à Constantinople pour participer à la vaine défense du Bosphore pour empêcher le passage des troupes ottomanes qui écrasèrent les forces terrestres des croisés à Varna, le 10 novembre. Au printemps suivant, il fut chargé d'explorer le rivage oriental de la mer Noire à la recherche de rescapés de Varna. Il alla en fait «a son adventure», capturant des

\footnotetext{
${ }^{50}$ Cf. D. Lalande, Jean II Le Meingre, dit Boucicaut (1366-1421). Étude d'une biographie hérö̈que, Genève (Publications romanes et françaises, CLXXXIV), 1988.

${ }^{51}$ Gutierre Díaz de Gamez, Le Victorial. Chronique de Don Pero Niño, comte de Buelna (1378-1453), trad. et éd. J. Gautier Dalché, Turnhout (Miroir du Moyen Âge), 2001, p. 125 160.

${ }^{52}$ Georges Chastellain, Euvres, éd. Kervyn de Lettenhove, t. V, Bruxelles, 1864 (reprint Genève, 1971), p. 46 (j'ai inversé les deux citations).

${ }^{53}$ Cf. mon livre La Politique navale des ducs de Bourgogne, 1384-1482, Lille (Économies et sociétés), 1995, p. 132, 138 et 139 ; nous ne savons ce qui se passa ensuite. Je prépare une étude sur ses lettres qui nous rapportent ces événements.
} 
navires turcs, dévastant le château d'Ünye, mais fut fait prisonnier sur les côtes de l'actuelle Géorgie en tentant de s'emparer d'un navire de chrétiens orientaux. Il fut libéré grâce à l'entremise de l'empereur de Trébizonde, reprit ses activités de pillage dans la mer Noire et la mer d'Azov jusqu'à Caffa. Obligé d'y vendre ses prises, il rentra auprès du duc de Bourgogne, alors que son cousin Jacot de Thoisy poursuivit une croisière offensive en mer Egée et en Méditerranée orientale jusqu'en 1447. De 1446 à 1449, Geoffroy de Thoisy dirigea à Anvers un chantier de construction de galères destinées à être envoyées dans le Levant. En 1454, il prononça le vœu de croisade au banquet du Faisan et fut un des responsables de la préparation navale de la croisade, toujours avec son cousin $\mathrm{Jacot}^{54}$.

Il est remarquable que peu de nobles semblent avoir profité de ces actions au service de la foi chrétienne pour devenir chevaliers. Les mentions, à ma connaissance, sont assez rares : Stephen Scrope à Alexandrie, en 1365, par Pierre I ${ }^{\mathrm{er}}$, roi de Chypre $^{55}$; Guillaume de Meuillon au siège devant Mahdia, en 1390, par Jean de Vienne, amiral de France ${ }^{56}$; Jean, comte de Nevers, avant l'attaque de la place bulgare de Vidin, en 1396, par Sigismond, roi de Hongrie; Geoffroy de Thoisy lors du siège de Rhodes, en $1444^{57}$. Remarquons pourtant que, sur sept écuyers français présents à la prise de Ceuta en 1415, trois devinrent chevaliers : Guy Le Boutiller, Martin de La Chapelle, Jacques de Liévin ${ }^{58}$. Il y avait un autre moyen, plus pacifique, de devenir chevalier: au Saint-Sépulcre, lors du pèlerinage aux Lieux saints $^{59}$.

Ayant accompli leurs hauts faits en Espagne, en Prusse, en Méditerranée orientale, les nobles ont-ils voulu en conserver la mémoire? Sans doute un certain nombre de relations d'expéditions de croisade durent être rédigés à leur suite et circuler assez largement, puis être intégrées par les chroniqueurs dans leurs œuvres. Un exemple a contrario est la Relation de la croisade de Nicopolis par un serviteur de Guy de Blois qui a repris le texte de Froissart. Geoffroy de Thoisy est probablement l'auteur d'un récit du siège de Rhodes et de ses aventures maritimes en 1444 et 1445 , en y incluant celles de son cousin Jacot jusqu'en $1447^{60}$, dont a pu se servir Jean Germain pour écrire son Liber de virtutibus (...) Philippi Burgundiae et Brabantiae ducis, à moins que celui-ci n'ait communiqué directement et verbalement avec Geoffroy de Thoisy.

\footnotetext{
${ }^{54} \mathrm{Cf}$. mes livres La Politique navale... et Les ducs de Bourgogne, la croisade et l'Orient..., en attendant mon étude sur le personnage.

${ }_{55}$ M. Keen, «Chaucer's Knight...», p. 52 ; A. Luttrell, «English Levantine crusaders...», p. 149 ; id., «Chaucer's Knight... », p. 142.

${ }^{56}$ Faits \& Gestes de Guillaume de Meuillon, éd. E. Magnin, Grenoble, 1897, p. 9.

${ }^{57}$ Cf. mon livre Les ducs de Bourgogne, la croisade et l'Orient..., p. 38 et 101.

${ }^{58}$ Cf. Antoine de La Sale, Le Reconfort de Madame de Fresne, éd. Ian Hill, Exeter (Textes littéraires, XXXIV), 1979, p. 27.

${ }^{59}$ Cf. J.-P. de Gennes, Les Chevaliers du Saint-Sépulcre de Jérusalem. Essai critique, t. I : Origines et histoire générale de l'Ordre, Cholet, 1995.

${ }^{60}$ Publié dans N. Iorga, «Les aventures "sarrazines" des Français de Bourgogne au XV siècle », Mélanges d'histoire générale, éd. Constantin Marinescu, Cluj (Université de Cluj, Publications de l'Institut d'histoire générale, I), 1927, doc. I, p. 30-35; j'en prépare une nouvelle édition.
} 
Individuellement, les nobles ont narré ou fait écrire, sur le même plan que leurs autres campagnes militaires, leurs expéditions de croisade dans leur biographie, ainsi Boucicaut dans le Livre des fais du bon messire Jehan le Maingre, dit Bouciquaut, mareschal de France et gouverneur de Jennes ${ }^{61}$, Pero Niño dans le Victorial écrit par son porte-bannière ${ }^{62}$, Guillaume de Meuillon dans ses Faits et Gestes $^{63}$, ou encore Georg von Ehingen dans ses souvenirs de voyages ${ }^{64}$. Plus original, Oswald von Wolkenstein a conservé en chansons le souvenir de ses expéditions : Prusse en 1399, Terre sainte en 1409-1410 (où il fut fait chevalier au Saint-Sépulcre), Ceuta en $1415^{65}$. Dans l'art, Alphonse V, roi de Portugal, fit réaliser des tapisseries pour commémorer ses prises d'Arzila et de Tanger en $1471^{66}$.

Pourtant l'image plus populaire du noble croisé de la fin du Moyen Âge qui reste est celle du Chevalier des Contes de Canterbury que Geoffroy Chaucer fait participer à la croisade d'Alexandrie, effectuer le voyage de Prusse contre les Lettons et les Russes, assister au siège d'Algeciras dans le royaume de Grenade, chevaucher (en tant que mercenaire) dans le royaume de Bellemarine (Maroc mérinide), être à la prise de L'Ayas et de Satalie, se trouver dans de nombreuses armées dans la Grande Mer (Méditerranée), se battre au service de l'émir de Balat (Milet) contre d'autres païens de Turquie..., image outrée, mais véridique dans les détails ${ }^{67}$. Il est vrai que dans leurs souvenirs, les nobles semblent mettre sur le même pied leurs expéditions de croisade et les autres campagnes militaires. Pour eux, comme dans les romans qu'ils lisaient, il semble que l'essentiel était d'accomplir des hauts faits d'armes : que ce fût en Prusse, en à Grenade, au Maroc, en Turquie ou en Égypte, ne faisait qu'ajouter une note d'exotisme. L'esprit de la croisade n'était pas mort, mais pour de nombreux nobles de l'Europe latine, il revêtait les habits de la fiction ${ }^{68}$.

\section{Jacques Paviot}

Université de Paris XII - Val de Marne

${ }^{61}$ Éd. D. Lalande, Paris-Genève (Textes littéraires français, 331), 1985 ; selon Hélène Millet, «Qui a écrit Le Livre des fais du bon messire Jehan Le Maingre dit Bouciquaut?» (dans Pratiques de la culture écrite en France au XV $V^{e}$ siècle, éd. Monique Ornato et Nicole Pons, Louvain-la-Neuve (Textes et études du Moyen Âge, 2), 1995, p. 135-149), l'auteur est Nicolas de Gonesse, confesseur du maréchal.

${ }^{62} \mathrm{Cf}$. référence supra.

${ }^{63} \mathrm{Cf}$. référence supra.

${ }^{64} \mathrm{Cf}$. référence supra.

${ }^{65}$ Cf. N. Mayr, Die Reiselieder und Reisen Oswalds von Wolkenstein, Innsbruck (SchlernSchriften, 215), 1961.

${ }^{66}$ Attribuées à un atelier tournaisien ; elles sont aussi dites de Pastrana, du nom de la ville où elles étaient conservées dans l'église ; elles semblent avoir été déplacées à L'Escurial.

${ }^{67} \mathrm{Cf}$. le livre de T. Jones, à corriger par les articles de M. Keen et d'A. Luttrell cités supra ; W. Paravicini, «Fahrende Ritter. Literarisches Bild und gelebte Wirklichkeit im Spätmittelalter», Mittelalterliche Menschenbilder, dir. M. Neumeyer, Regensburg (Eichstätter Kolloquium, 8), 2000, p. 205-254.

${ }^{68}$ Il est remarquable que Geoffroy de Thoisy a pu être un modèle de Tirant le Blanc; cf. C. Marinesco, « Du nouveau sur Tirant lo Blanch », Estudis romànics, IV, 1953-1954, p. 137203. 